\title{
Analysis of Iterative Learning Control for a Class of Linear Discrete-Time Switched Systems
}

\author{
Xuan Yang and Xiaoe Ruan \\ Department of Applied Mathematics, School of Mathematics and Statistics, Xi'an Jiaotong University, No. 28, \\ Xianning West Road, Xian, Shaanxi 710049, China
}

Correspondence should be addressed to Xuan Yang; yangxuan@stu.xjtu.edu.cn

Received 12 September 2014; Accepted 19 December 2014

Academic Editor: Hamid Reza Karimi

Copyright (C) 2015 X. Yang and X. Ruan. This is an open access article distributed under the Creative Commons Attribution License, which permits unrestricted use, distribution, and reproduction in any medium, provided the original work is properly cited.

An iterative learning control scheme is applied to a class of linear discrete-time switched systems with arbitrary switching rules. The application is based on the assumption that the switched system repetitively operates over a finite time interval. By taking advantage of the super vector approach, convergence is discussed when noise is free and robustness is analyzed when the controlled system is disturbed by bounded noise. The analytical results manifest that the iterative learning control algorithm is feasible and effective for the linear switched system. To support the theoretical analysis, numerical simulations are made.

\section{Introduction}

A switched system consists of a family of subsystems described by differential equations or difference equations, whose switching rules are usually considered to be arbitrary. The switched systems belong to the hybrid systems and have attracted flourishing investigations in the latest decade [1-8]. The current study of the switched system focuses mainly on the analysis of the dynamic behaviors including stability, controllability, reachability, and observability [1-6]. In particular, the concept of finite time stochastic stability for switched stochastic systems under asynchronous switching assumption has been raised as shown in recent references [7, 8]. The basic reason for studying such systems comes mainly from the fact that the switched systems have been widely emerged in the field of control and engineering practice, such as chemical systems, process control, and automotive industry $[9,10]$. Although the investigation on the switched systems is flourishing, the study on designing a controller for trajectory tracking of the switched system is still rare. On the other hand, in engineering practice, the dynamics of the plant model is usually unknown and some uncertain disturbance is unavoidable. These adverse effects together with random switching rules increase challenge of designing an efficacious controller for a tracking performance of a switched system.
Fortunately, there exists a kind of efficient control scheme, named as iterative learning control (ILC), which has also drawn increasing attention for its simple control structure and perfect learning performance. One of the advantages of ILC is that it requires less knowledge of the controlled system in the procedure of learning. It utilizes the tracking error information of the previous operations to compensate for the current control input so as to generate an upgraded control input for the next operation. By this successive learning process, the tracking performance of the controlled system is improved. In view of the above properties of ILC, it can be used in the switched systems for tracking a given target. However, to authors' knowledge, there are few efforts on the study of the ILC strategies for the switched systems. So far, few literatures $[9,11,12]$ have focused on the issue.

In literature [9], a P-type ILC scheme has been applied to a class of linear discrete-time switched systems. The convergence property for such an ILC system has been analyzed by super vector approach. However, the robustness of the ILC algorithm is not covered for the case when the controlled system is interfered by model uncertainty or external noise. As known, there exist two main criteria to measure the learning performance of an ILC scheme. One is convergence and the other is robustness. Since noise and uncertainty 
are inevitable in the engineering practice, it is necessary to analyze the robustness of an ILC algorithm.

In the literatures $[11,12]$, D-type ILC algorithms have been proposed for a class of discrete-time linear and nonlinear switched systems. The convergence conditions are derived in the sense of $\lambda$-norm. Yet, it has been commented that, in the literature [13], when using $\lambda$-norm, the impact of the system dynamics and the learning gains on the learning performance is extremely suppressed by the sufficiently larger parameter $\lambda$ to ensure the convergence. Thus it is possible that an improper choice of the parameter $\lambda$ may spoil a fair assessment of the tracking behaviors in practice [13]. Also, the articles [11,12] do not involve the robustness which is a key criterion of learning algorithm. Thus the study of ILC algorithms for the switched systems needs to be further expanded.

Motivated by the drawbacks of the literatures $[9,11,12]$, the paper discusses the convergence and the robustness of a $\mathrm{P}$ type ILC algorithm for a kind of discrete linear discrete timeinvariant switched systems with a fixed arbitrary switching rule. The convergence and robustness are analyzed in the iteration domain using the super vector approach. Firstly, the sufficient condition of convergence is given and then, significantly, the robustness of the algorithm is analyzed when the control switched system is interfered by bounded measurement noise. In order to manifest the validity and effectiveness of the ILC algorithm, numerical simulation for a simple example is made.

The rest of the paper is organized as follows. Section 2 presents the fundamental problem. Section 3 exhibits the learning process description in the form of super vector and the learning performance analysis. In order to show the feasibility and effectiveness of the theoretical results, numerical simulations are given in Section 4 and the conclusion is drawn in the last section.

\section{Fundamental Problem Description}

Consider a class of linear discrete time-invariant single-input, single-output switched systems described as follows:

$$
\begin{aligned}
& \mathbf{x}_{k+1}(i+1)=\mathbf{A}_{\tau(i)} \mathbf{x}_{k+1}(i)+\mathbf{B}_{\tau(i)} u_{k+1}(i), \\
& y_{k+1}(i+1)=\mathbf{C}_{\tau(i)} \mathbf{x}_{k+1}(i+1)
\end{aligned}
$$

$$
i \in S \text {. }
$$

Here, $\mathbf{x}_{k+1}(i) \in \mathbb{R}^{n}, u_{k+1}(i) \in \mathbb{R}$, and $y_{k+1}(i+1) \in \mathbb{R}$ are the state variable, the input variable, and the output variable, respectively. The set $S=\{0,1,2, \ldots, N-1\}, N \in \mathbb{Z}_{+}$, stands for the operation time duration each trial, $i ; i \in S$ denotes the time instant. The subscript $k+1, k \in \mathbb{N}$, denotes iteration number. The symbol $\tau(i)$ represents random switching rule defined by $\tau(\cdot): \mathbb{N} \rightarrow Q=\{1,2,3, \ldots, q\}$ with $q \in \mathbb{Z}_{+}, q<$ $+\infty$. This implies that the matrices group $\left(\mathbf{A}_{\tau(i)}, \mathbf{B}_{\tau(i)}, \mathbf{C}_{\tau(i)}\right)$ can be taken as an arbitrary element of following finite set:

$$
\left\{\left(\mathbf{A}_{1}, \mathbf{B}_{1}, \mathbf{C}_{1}\right),\left(\mathbf{A}_{2}, \mathbf{B}_{2}, \mathbf{C}_{2}\right), \ldots,\left(\mathbf{A}_{q}, \mathbf{B}_{q}, \mathbf{C}_{q}\right)\right\} \text {. }
$$

For a given desired trajectory $y_{d}(i+1), i \in S$, the objective of an ILC for the system (1) is to generate an input sequence $\left\{u_{k}(i), i \in S, k \in \mathbb{Z}_{+}\right\}$in a recursive mode by learning from the previous experience such that it can drive the system (1) to track the desired trajectory $y_{d}(i+1), i \in S$, as precisely as possible, mathematically:

$$
\lim _{k \rightarrow \infty}\left|e_{k+1}(i+1)\right|=0, \quad \forall i \in S,
$$

where $e_{k+1}(i+1)=y_{d}(i+1)-y_{k+1}(i+1)$ is the tracking error.

Analysis of this paper is basis of the following primary assumptions $[9,11]$.

(A1) All operations start at identical initial state; that is, $\mathbf{x}_{k+1}(0)=\mathbf{x}_{0}$, for any $k \in \mathbb{Z}_{+}$. Without loss of generality, it is assumed that $\mathbf{x}_{0}=\mathbf{0}$.

(A2) The desired trajectory $y_{d}(i+1), i \in S$, is iterationinvariant.

(A3) For the given desired trajectory $y_{d}(i+1), i \in S$, there exists a desired control input signal $u_{d}(i), i \in S$, and a desired state vector $\mathbf{x}_{d}(i), i \in S$, such that

$$
\begin{aligned}
& \mathbf{x}_{d}(i+1)=\mathbf{A}_{\tau(i)} \mathbf{x}_{d}(i)+\mathbf{B}_{\tau(i)} u_{d}(i), \\
& y_{d}(i+1)=\mathbf{C}_{\tau(i)} \mathbf{x}_{d}(i+1)
\end{aligned}
$$

$i \in S$.

In this paper, a P-type ILC scheme is considered as

$$
u_{k+1}(i)=u_{k}(i)+\gamma_{\tau(i)} e_{k}(i+1),
$$

where $\gamma_{\tau(i)}(\tau(i) \in Q)$ denotes the proportional learning gain.

\section{Learning Performances Analysis}

In this section, the super vector approach is employed to analyze the learning performance of the algorithm (5) for the switched systems (1).

3.1. Super Vector Representation of ILC. Given an arbitrary $i \epsilon$ $S$, the outputs at time instants from 1 to $i+1$ of the $(k+1)$ th trial can be, respectively, calculated as

$$
\begin{aligned}
y_{k+1}(1)= & \mathbf{C}_{\tau(0)} \mathbf{x}_{k+1}(1) \\
= & \mathbf{C}_{\tau(0)} \mathbf{A}_{\tau(0)} \mathbf{x}_{k+1}(0)+\mathbf{C}_{\tau(0)} \mathbf{B}_{\tau(0)} u_{k+1}(0), \\
y_{k+1}(2)= & \mathbf{C}_{\tau(1)} \mathbf{x}_{k+1}(2) \\
= & \mathbf{C}_{\tau(1)} \mathbf{A}_{\tau(1)} \mathbf{A}_{\tau(0)} \mathbf{x}_{k+1}(0) \\
& +\mathbf{C}_{\tau(1)} \mathbf{A}_{\tau(1)} \mathbf{B}_{\tau(0)} u_{k+1}(0)+\mathbf{C}_{\tau(1)} \mathbf{B}_{\tau(1)} u_{k+1}(1), \\
y_{k+1}(3)= & \mathbf{C}_{\tau(2)} \mathbf{x}_{k+1}(3) \\
= & \mathbf{C}_{\tau(2)} \mathbf{A}_{\tau(2)} \mathbf{A}_{\tau(1)} \mathbf{A}_{\tau(0)} \mathbf{x}_{k+1}(0) \\
& +\mathbf{C}_{\tau(2)} \mathbf{A}_{\tau(2)} \mathbf{A}_{\tau(1)} \mathbf{B}_{\tau(0)} u_{k+1}(0) \\
& +\mathbf{C}_{\tau(2)} \mathbf{A}_{\tau(2)} \mathbf{B}_{\tau(1)} u_{k+1}(1)+\mathbf{C}_{\tau(2)} \mathbf{B}_{\tau(2)} u_{k+1}(2),
\end{aligned}
$$




$$
\begin{aligned}
y_{k+1}(i+1)= & \mathbf{C}_{\tau(i)} \mathbf{x}_{k+1}(i+1) \\
= & \mathbf{C}_{\tau(i)} \prod_{j=0}^{i} \mathbf{A}_{\tau(j)} \mathbf{x}_{k+1}(0) \\
& +\mathbf{C}_{\tau(i)} \prod_{j=1}^{i} \mathbf{A}_{\tau(j)} \mathbf{B}_{\tau(0)} u_{k+1}(0) \\
& +\mathbf{C}_{\tau(i)} \prod_{j=2}^{i} \mathbf{A}_{\tau(j)} \mathbf{B}_{\tau(1)} u_{k+1}(1) \\
& +\cdots+\mathbf{C}_{\tau(i)} \mathbf{A}_{\tau(i)} \mathbf{B}_{\tau(i-1)} u_{k+1}(i-1) \\
& +\mathbf{C}_{\tau(i)} \mathbf{B}_{\tau(i)} u_{k+1}(i)
\end{aligned}
$$

For the sake of expression simplicity, the following "super vectors" are denoted as

$$
\begin{aligned}
& \mathbf{u}_{k+1}=\left[u_{k+1}(0), u_{k+1}(1), \ldots, u_{k+1}(i)\right]^{\mathrm{T}}, \\
& \mathbf{y}_{k+1}=\left[y_{k+1}(1), y_{k+1}(2), \ldots, y_{k+1}(i+1)\right]^{\mathrm{T}} .
\end{aligned}
$$

Then the system (6) can be equivalently represented as the following input-output response linear system:

$$
\mathbf{y}_{k+1}=\mathbf{H}(i) \mathbf{u}_{k+1}+\mathbf{D}(i)
$$

(6)

where

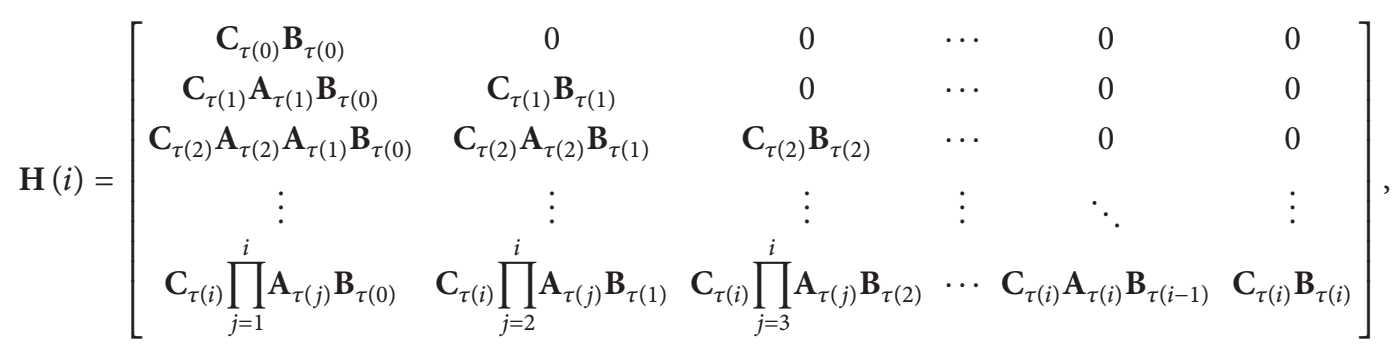

$$
\begin{aligned}
& \mathbf{D}(i)=\left[\mathbf{C}_{\tau(0)} \mathbf{A}_{\tau(0)} x_{k+1}(0), \mathbf{C}_{\tau(1)} \prod_{j=0}^{1} \mathbf{A}_{\tau(j)} x_{k+1}(0), \mathbf{C}_{\tau(2)} \prod_{j=0}^{2} \mathbf{A}_{\tau(j)} x_{k+1}(0), \ldots, \mathbf{C}_{\tau(i)} \prod_{j=0}^{i} \mathbf{A}_{\tau(j)} x_{k+1}(0)\right]^{\mathbf{T}} .
\end{aligned}
$$

Taking assumption (A1) into account reduces $\mathbf{D}(i)=\mathbf{0}$. Considering $i=N-1$ and denoting $\mathbf{H}(N-1)=\mathbf{H}$, the system (8) becomes

$$
\mathbf{y}_{k+1}=\mathbf{H} \mathbf{u}_{k+1} \text {, }
$$

where $\mathbf{u}_{k+1}=\left[u_{k+1}(0), u_{k+1}(1), \ldots, u_{k+1}(N-1)\right]^{\mathrm{T}}$ and $\mathbf{y}_{k+1}=$ $\left[y_{k+1}(1), y_{k+1}(2), \ldots, y_{k+1}(N)\right]^{\mathrm{T}}$.

Remark 1. It is noted that the presentation in the form of super vector reflects the dynamical properties of the system (1) in the iteration domain. For the linear time-varying switched systems and some kinds of nonlinear switched systems, similar description can also be derived. Thus the results in this paper can be generalized to some linear timevarying switched systems and some classes of nonlinear switched systems.

Consequently, the control objective of ILC in the form of super vector can be equivalently described as searching such an input super vector sequence $\left\{\mathbf{u}_{k}\right\}, k \in \mathbb{Z}_{+}$that it can drive the system (10) to track the desired trajectory $\mathbf{y}_{d}$ as precisely as possible as the iteration number tends to infinite; that is,

$$
\lim _{k \rightarrow \infty}\left\|\mathbf{e}_{k+1}\right\|_{\nu}=0
$$

Here, “\| $\cdot \|_{\gamma}$ " denotes some vector norm and $\mathbf{e}_{k+1}$ represents tracking error super vector which is defined as

$$
\begin{gathered}
\mathbf{e}_{k+1}=\mathbf{y}_{d}-\mathbf{y}_{k+1}=\left[e_{k+1}(1), e_{k+1}(2), \ldots, e_{k+1}(N)\right]^{\mathrm{T}}, \\
\mathbf{y}_{d}=\left[y_{d}(1), y_{d}(2), \ldots, y_{d}(N)\right]^{\mathrm{T}} .
\end{gathered}
$$

In the form of the super vector, the updating law (5) is reformulated as

$$
\mathbf{u}_{k+1}=\mathbf{u}_{k}+\Gamma \mathbf{e}_{k}
$$

where

$$
\boldsymbol{\Gamma}=\left[\begin{array}{ccccc}
\gamma_{\tau(0)} & 0 & 0 & \cdots & 0 \\
0 & \gamma_{\tau(1)} & 0 & \cdots & 0 \\
0 & 0 & \gamma_{\tau(2)} & \cdots & 0 \\
\vdots & \vdots & \vdots & \ddots & \vdots \\
0 & 0 & 0 & \cdots & \gamma_{\tau(N-1)}
\end{array}\right]
$$

is the learning gain matrix which consists of the learning gains of updating law (5). Now original two-dimensional ILC problem is converted into a one-dimensional linear inputoutput response problem [14]. 


\subsection{Convergence and Robustness Analysis}

Theorem 2. Assume that the ILC algorithm (13) is imposed on the linear switched system (10) with an arbitrary switching sequence $\{\tau(i)\}, i \in S$. Then the tracking error converges monotonically in the sense of some vector norm if the inequality

$$
\|\mathbf{I}-\mathbf{H} \Gamma\|_{\mu}<1
$$

holds, where " \|\|$_{\mu}$ " denotes the induced matrix norm.

Proof. By the definition of the tracking error, it is easy to derive

$$
\begin{aligned}
\mathbf{e}_{k+1} & =\mathbf{y}_{d}-\mathbf{y}_{k+1} \\
& =\mathbf{y}_{d}-\mathbf{H}\left(\mathbf{u}_{k}+\Gamma \mathbf{e}_{k}\right) \\
& =(\mathbf{I}-\mathbf{H} \Gamma) \mathbf{e}_{k} .
\end{aligned}
$$

Taking norm of both sides of (16) and using norm inequality yield

$$
\left\|\mathbf{e}_{k+1}\right\|_{\nu} \leq\|\mathbf{I}-\mathbf{H} \Gamma\|_{\mu}\left\|\mathbf{e}_{k}\right\|_{\nu},
$$

where $v=1,2$ or $\infty$. Considering assumption $\|\mathbf{I}-\mathbf{H} \Gamma\|_{\mu}<1$ gets

$$
\left\|\mathbf{e}_{k+1}\right\|_{\nu}<\left\|\mathbf{e}_{k}\right\|_{\nu} .
$$

This completes the proof.

It is noted that the elements of the matrix $\mathbf{H}$ are not determined in advance, as the switching rule of the controlled system is arbitrary. In the process of analysis, it is assumed that the condition (15) holds for all possibilities of $\mathbf{H}$.

Remark 3. The assumption (15) is a sufficient condition for monotone convergence. In particular, in the case when $\mu=2$, $\nu=2$, it derives

$$
\begin{aligned}
\left\|\mathbf{e}_{k+1}\right\|_{2} & \leq\|\mathbf{I}-\mathbf{H} \boldsymbol{\Gamma}\|_{2}\left\|\mathbf{e}_{k}\right\|_{2}=\sqrt{\rho_{\max }(\mathbf{I}-\mathbf{H} \boldsymbol{\Gamma})^{\mathrm{T}}(\mathbf{I}-\mathbf{H} \boldsymbol{\Gamma})}\left\|\mathbf{e}_{k}\right\|_{2} \\
& =\sigma_{\max }(\mathbf{I}-\mathbf{H} \boldsymbol{\Gamma})\left\|\mathbf{e}_{k}\right\|_{2} .
\end{aligned}
$$

Here $\rho(\mathbf{M})$ denotes the spectral radius of $\mathbf{M}$ and $\sigma(\mathbf{M})$ represents the singular value of $\mathbf{M}$, which is defined as $\sigma(\mathbf{M})=$ $\sqrt{\rho\left(\mathbf{M}^{\mathrm{T}} \mathbf{M}\right)}$. In the following discussion, we consider that $\mu=$ $2, v=2$ in (15).

When considering measurement disturbance $\xi_{k+1}(i+1)$, $i \in S$, the system (1) can be reformulated as

$$
\begin{array}{r}
\mathbf{x}_{k+1}(i+1)=\mathbf{A}_{\tau(i)} \mathbf{x}_{k+1}(i)+\mathbf{B}_{\tau(i)} u_{k+1}(i), \\
y_{k+1}(i+1)=\mathbf{C}_{\tau(i)} \mathbf{x}_{k+1}(i+1)+\xi_{k+1}(i+1) \\
i \in S .
\end{array}
$$

Thus the system (10) can be reformulated as

$$
\mathbf{y}_{k+1}=\mathbf{H} \mathbf{u}_{k+1}+\boldsymbol{\xi}_{k+1},
$$

where $\boldsymbol{\xi}_{k+1}=\left[\xi_{k+1}(1), \xi_{k+1}(2), \ldots, \xi_{k+1}(N)\right]^{\mathrm{T}}$ denotes the measurement noise vector satisfying $\left\|\xi_{k+1}\right\|_{2}<+\infty$ for any $k \in \mathbb{Z}_{+}$.

There are two types of errors, namely, the contaminated tracking error $\overline{\mathbf{e}}_{k+1}$ and the uncontaminated tracking error $\mathbf{e}_{k+1}$ to measure the learning behavior at the $(k+1)$ th trial. They are, respectively, defined as

$$
\begin{aligned}
& \overline{\mathbf{e}}_{k+1}=\mathbf{y}_{d}-\mathbf{G u}_{k+1}-\boldsymbol{\xi}_{k+1}, \\
& \mathbf{e}_{k+1}=\mathbf{y}_{d}-\mathbf{G} \mathbf{u}_{k+1} .
\end{aligned}
$$

Clearly, the relationship of $\mathbf{e}_{k+1}$ and $\overline{\mathbf{e}}_{k+1}$ is $\mathbf{e}_{k+1}=\overline{\mathbf{e}}_{k+1}+\boldsymbol{\xi}_{k+1}$.

The signal $\mathbf{e}_{k+1}$ is the observed error at the $(k+1)$ th iteration regardless of the noise at this trial (it is only influenced by previous noise signals $\left.\xi_{l}, 0 \leq l \leq k\right)$. It is an option to assess the tracking behavior of the learning algorithm, whereas, since the noise is transferred by the learning process, the current input vector $\mathbf{u}_{k}$ ought to be compensated by the contaminated error $\overline{\mathbf{e}}_{k}$ at the $k$ th trial; that is to say, the updating law (13) is reformulated as

$$
\mathbf{u}_{k+1}=\mathbf{u}_{k}+\Gamma \overline{\mathbf{e}}_{k} .
$$

By the definition of the tracking error vector, the evolution of the uncontaminated tracking error vector at two adjacent trials can be inferred as

$$
\mathbf{e}_{k+1}=(\mathbf{I}-\mathbf{H} \Gamma) \mathbf{e}_{k}+\mathbf{H} \Gamma \boldsymbol{\xi}_{k}
$$

Theorem 4. Consider (24) which is derived by using the updating law (23) on the switched system (21). Then $\lim _{k \rightarrow \infty}$ sup $\left\|\mathbf{e}_{k+1}\right\|_{2}$ is bounded if the noise vectors satisfy $\left\|\boldsymbol{\xi}_{k}\right\|_{2}=\beta<+\infty$ for any $k \in \mathbb{Z}_{+}$and $\sigma_{\max }(\mathbf{I}-\mathbf{H} \boldsymbol{\Gamma})=\bar{\sigma}<1$, where "sup" stands for the supremum of a sequence.

Proof. Taking the Euclidean norm of both sides of (24) yields

$$
\begin{aligned}
\left\|\mathbf{e}_{k+1}\right\|_{2} & \leq\|(\mathbf{I}-\mathbf{H} \Gamma)\|_{2}\left\|\mathbf{e}_{k}\right\|_{2}+\|\mathbf{H} \Gamma\|_{2}\left\|\boldsymbol{\xi}_{k}\right\|_{2} \\
& \leq\|(\mathbf{I}-\mathbf{H} \Gamma)\|_{2}\left\|\mathbf{e}_{k}\right\|_{2}+\|\mathbf{H} \Gamma\|_{2} \beta .
\end{aligned}
$$

Using the relation (25) successively derives

$$
\begin{aligned}
& \left\|\mathbf{e}_{k+1}\right\|_{2} \leq\|(\mathbf{I}-\mathbf{H} \Gamma)\|_{2}\left\|\mathbf{e}_{k}\right\|_{2}+\|\mathbf{H} \boldsymbol{\Gamma}\|_{2} \beta \\
& \leq\|(\mathbf{I}-\mathbf{H} \Gamma)\|_{2}\left[\|(\mathbf{I}-\mathbf{H} \boldsymbol{\Gamma})\|_{2}\left\|\mathbf{e}_{k-1}\right\|_{2}+\|\mathbf{H} \Gamma\|_{2} \beta\right] \\
& +\|\mathbf{H} \boldsymbol{\Gamma}\|_{2} \beta \\
& =\|(\mathbf{I}-\mathbf{H} \boldsymbol{\Gamma})\|_{2}^{2}\left\|\mathbf{e}_{k-1}\right\|_{2}+\left(\|(\mathbf{I}-\mathbf{H} \boldsymbol{\Gamma})\|_{2}+1\right)\|\mathbf{H} \boldsymbol{\Gamma}\|_{2} \beta \\
& \leq\|(\mathbf{I}-\mathbf{H} \Gamma)\|_{2}^{k}\left\|\mathbf{e}_{1}\right\|_{2}+\left(\sum_{j=0}^{k-1}\|(\mathbf{I}-\mathbf{H} \boldsymbol{\Gamma})\|_{2}^{j}\right)\|\mathbf{H} \Gamma\|_{2} \beta .
\end{aligned}
$$


Taking the assumption $\left\|\boldsymbol{\xi}_{k}\right\|_{2}=\beta<+\infty$ for any $k \in \mathbb{Z}_{+}$into account and denoting $\bar{\sigma}=\sigma_{\max }(\mathbf{I}-\mathbf{H} \Gamma)$, inequality (26) becomes

$$
\begin{aligned}
\left\|\mathbf{e}_{k+1}\right\|_{2} & \leq \bar{\sigma}^{k}\left\|\mathbf{e}_{1}\right\|_{2}+\left(\sum_{j=0}^{k-1} \bar{\sigma}^{j}\right)\|\mathbf{H} \boldsymbol{\Gamma}\|_{2} \beta \\
& =\bar{\sigma}^{k}\left\|\mathbf{e}_{1}\right\|_{2}+\frac{1-\bar{\sigma}^{k}}{1-\bar{\sigma}}\|\mathbf{H} \Gamma\|_{2} \beta .
\end{aligned}
$$

Since $\bar{\sigma}<1$, it is easy to conclude that $\left(1-\bar{\sigma}^{k}\right) /(1-\bar{\sigma})<$ $\left(1-\bar{\sigma}^{k-1}\right) /(1-\bar{\sigma})$. This implies that

$$
\begin{aligned}
& \lim _{k \rightarrow \infty} \sup \left\|\mathbf{e}_{k+1}\right\|_{2} \\
& \quad \leq \lim _{k \rightarrow \infty} \bar{\sigma}^{k}\left\|\mathbf{e}_{1}\right\|_{2}+\lim _{k \rightarrow \infty} \sup \left(\frac{1-\bar{\sigma}^{k}}{1-\bar{\sigma}}\right)\|\mathbf{H} \boldsymbol{\Gamma}\|_{2} \beta \\
& \quad=\frac{\|\mathbf{H} \boldsymbol{\Gamma}\|_{2} \beta}{1-\bar{\sigma}} .
\end{aligned}
$$

This completes the proof.

Remark 5. It is seen from estimation (28) that the value of $\lim _{k \rightarrow \infty} \sup \left\|\mathbf{e}_{k+1}\right\|_{2}$ is mainly related to the boundary $\beta$ of the noise. The smaller magnitude of $\beta$ will lead to better tracking performance. In particular, when the noise is free, the monotone convergence of the uncontaminated tracking error in the sense of Euclidean norm is achieved, which is consistent with Theorem 2 . In the case when the value of $\beta$ is determined, the influence of the noise can be reduced by decreasing the value of $\sigma(\mathbf{I}-\mathbf{H} \mathbf{\Gamma})$.

Remark 6. The super vector approach employed in the paper is a tool to theoretically analyze the learning performance of the addressed ILC algorithm. Since it converts the original two-dimensional problem into a one-dimension linear inputoutput response problem, it can deal with the ILC problem only in the iterative domain regardless of time domain. This brings a great convenience for designing a controller and for theoretical analysis.

Remark 7. Compared with the existing ILC algorithms for the linear switched systems in the literatures [9, 11], this paper analyzes robustness to external measurement noise by employing super vector method, which is one of significant issues in the procedure of designing an applicable controller. Additionally, it is observed that, for the switched systems, the convergence and robustness are guaranteed on the basis that the system dynamics is required to be iteration-invariant, though the system arbitrarily switches among the subdynamics. The case that can be regarded as the system dynamics is time-varying. The analysis implies that the ILC scheme may work well for time-varying systems. This turns to be the significance of the ILC strategy that it requires less a prior of system knowledge.

Remark 8. It is necessary to point out that the study on the ILC algorithms for switched systems is more arduous than that for nonswitched systems, since the dynamical behaviors of switched systems are more complex. As seen that a switched system obeys diverse subdynamics, compared with an ILC algorithm for a nonswitched system, the ILC scheme for a switched system requires much more memory to store the switching rules as well as the subdynamics. It would be a dimensional disaster in the case when the system dimension is higher and the sampling number is much larger. However, the memory requirement will be solved sooner or later with the advancing of information technology. Next, hinted by the manner of the existing references $[9,11,12,15]$, the study of the paper for the switched system is based on the premise that the switching rule is time-dependent but iteration-invariant; that is, the switching rule varies along the time axis but it must be fixed for the whole learning process once the switching rule is selected for the starting learning. This requirement is crucial. For a general case when the switching rule is fired randomly both in time axis and in iteration direction, how to analyze the learning performance is a key difficulty. The issue will be investigated in future work.

\section{Numerical Simulations}

To manifest the validity and the effectiveness of the algorithm, a simple example is considered, which has served as an example in the literature [9]. The switched system with three subsystems is formulated as follows:

$$
\begin{aligned}
& \mathbf{x}_{k+1}(i+1)=\mathbf{A}_{\tau(i)} \mathbf{x}_{k+1}(i)+\mathbf{B}_{\tau(i)} u_{k+1}(i), \\
& y_{k+1}(i+1)=\mathbf{C}_{\tau(i)} \mathbf{x}_{k+1}(i+1)
\end{aligned}
$$

$i \in S$.

Here, the operation discrete time duration is set as $S=$ $\{0,1,2, \ldots, 59\}$ (i.e., $N=60$ ). Given arbitrary positive integers $v_{i}(i \in S)$ belonging to $[1,60]$, the arbitrarily switching sequence is defined as

$$
\tau(i)= \begin{cases}1, & \text { if } v_{i} \bmod 3=1 \\ 2, & \text { if } v_{i} \bmod 3=2 \\ 3, & \text { if } v_{i} \bmod 3=0\end{cases}
$$

Two possible switching sequences of $\tau(i)$ are shown in Figure 1.

The system dynamics are given as

$$
\begin{aligned}
& \mathbf{A}_{1}=\left[\begin{array}{cc}
0 & 1 \\
0.125 & -0.2
\end{array}\right], \quad B_{1}=\left[\begin{array}{l}
0 \\
1
\end{array}\right], \quad C_{1}=\left[\begin{array}{ll}
0.1 & 1
\end{array}\right], \\
& \mathbf{A}_{2}=\left[\begin{array}{cc}
-0.25 & 1 \\
0 & -0.3
\end{array}\right], \quad B_{2}=\left[\begin{array}{l}
0 \\
1
\end{array}\right], \quad C_{2}=\left[\begin{array}{ll}
-0.2 & 1
\end{array}\right] \text {, } \\
& \mathbf{A}_{3}=\left[\begin{array}{cc}
1 & 0 \\
0.2 & -0.1
\end{array}\right], \quad B_{3}=\left[\begin{array}{l}
0 \\
1
\end{array}\right], \quad C_{3}=\left[\begin{array}{ll}
0.25 & 1
\end{array}\right] \text {. }
\end{aligned}
$$

The desired trajectory is selected as $y_{d}(i+1)=\sin (8 i / 25)$, $i \in S$. The initial state is set as $\mathbf{x}_{k+1}(0)=\mathbf{0}$ for all $k \in \mathbb{N}$ and the starting input vector is chosen as $\mathbf{u}_{1}(0)=\mathbf{0}$. Throughout 

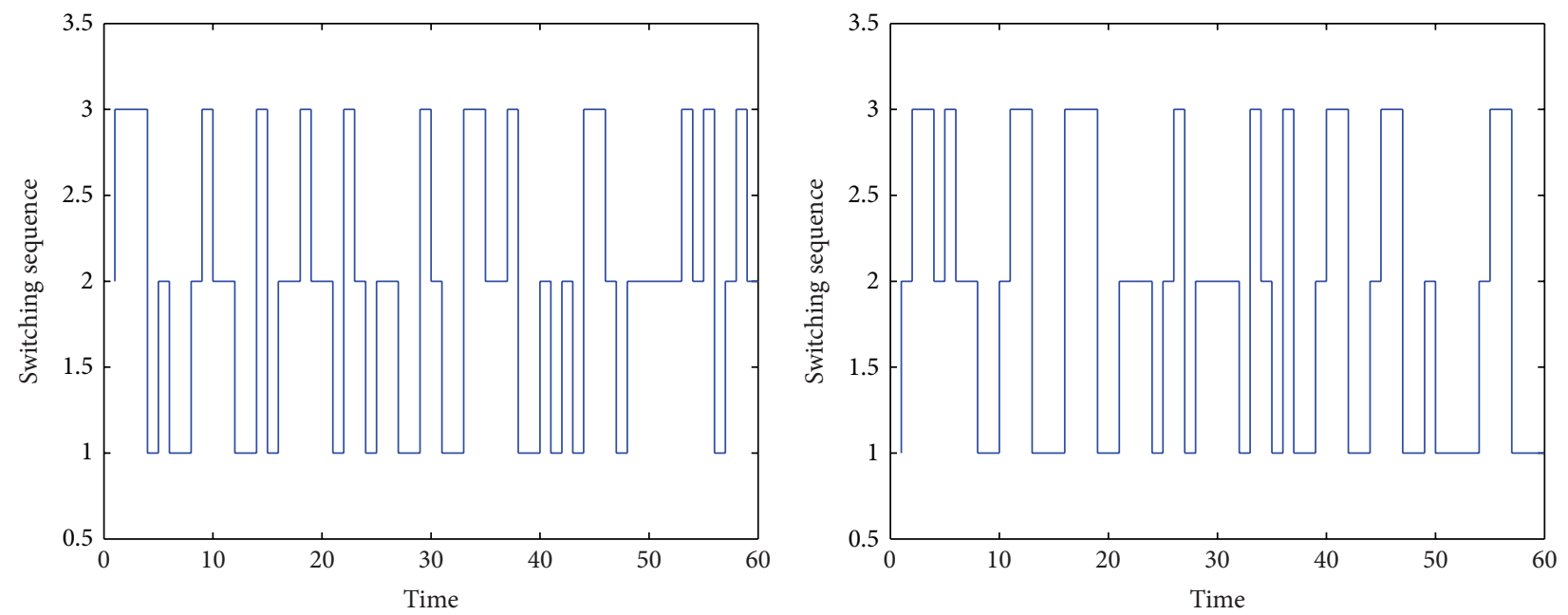

FIGURE 1: Arbitrary switching sequence $\tau(i)$.

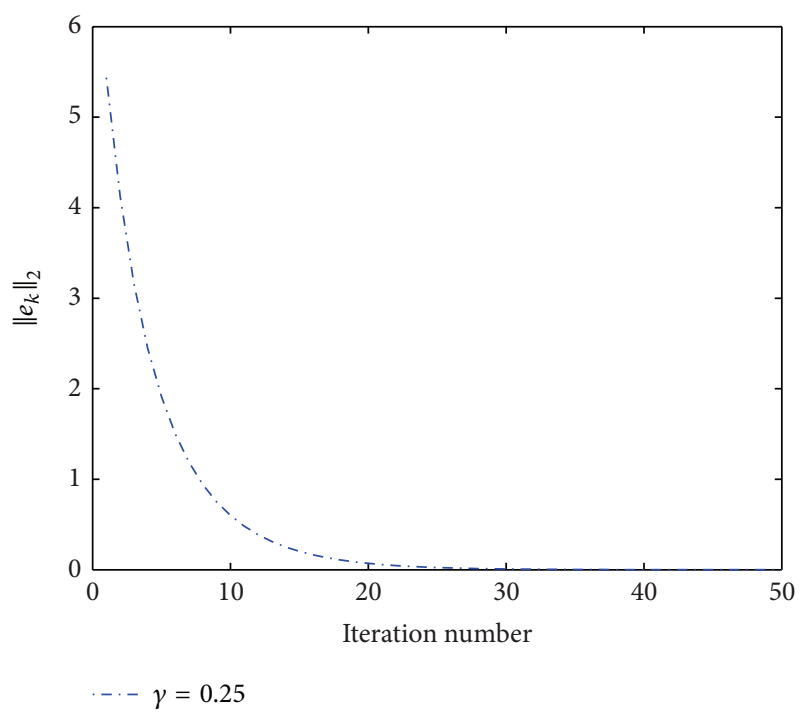

Figure 2: Tracking error in iteration domain.

the implementation, the learning gains are chosen to be timeinvariant and set as $\gamma_{\tau(i)}=0.25$. In this case, the condition $\|\mathbf{I}-\mathbf{H} \Gamma\|_{2}=\bar{\sigma}=0.8475<1$ holds.

4.1. Convergence. Assume that the system (29) is not interfered by any external noise and the updating law (13) is applied. Figure 2 exhibits the curve of $\left\|\mathbf{e}_{k}\right\|_{2}$ from the 1st iteration to the 50th iteration. Obviously, $\left\|\mathbf{e}_{k}\right\|_{2}$ is convergent in the iteration domain. Figure 3 depicts the system outputs at the 5th iteration, the 10th iteration, and the 15 th iteration. It is seen that the output of the switched system (29) can track the desired trajectory asymptotically as the iteration number enlarges. These indicate that ILC algorithm (13) is valid for the case when uncertain noise is neglected.

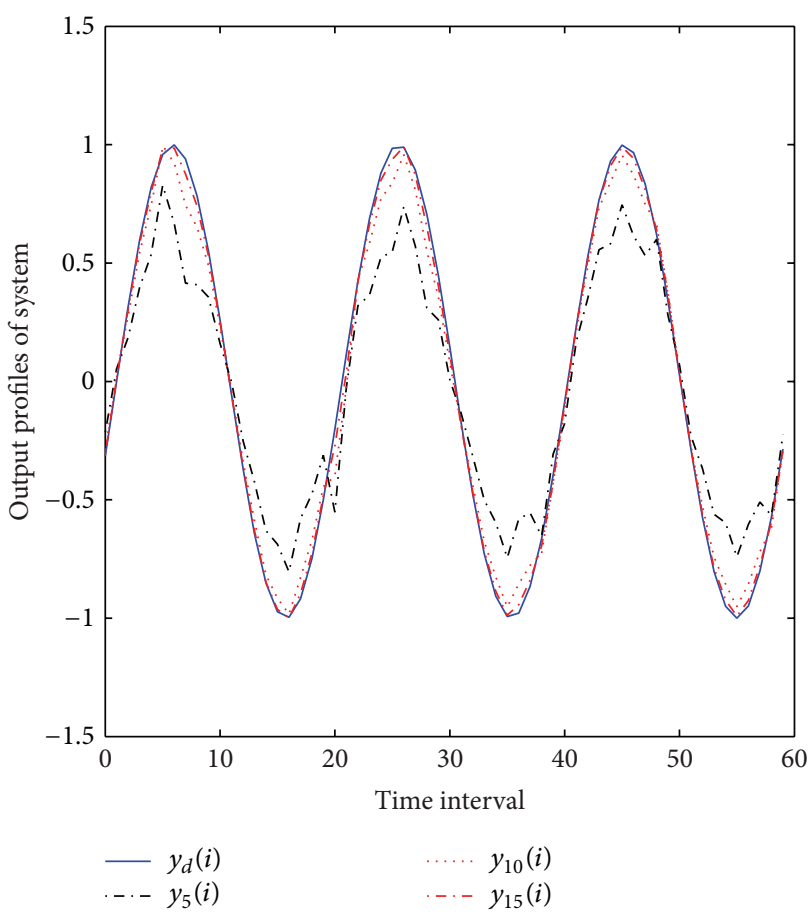

FIgURE 3: The system outputs at the 5th trial, the 10th trial, and the 15 th trial.

4.2. Robustness. Suppose that the system (29) is randomly disturbed by a set of bounded measurement disturbance. One of disturbance set in an arbitrary trial is shown in Figure 4. The boundary of $\sup \left(\left\|\mathbf{e}_{k+1}\right\|_{2}\right)$ implies that $\left\|\mathbf{e}_{k+1}\right\|_{2}$ is bounded too. The value of $\left\|\mathbf{e}_{k}\right\|_{2}$ is calculated and its profile is shown in Figure 5. Then the magnitude of $\max \left(\left\|\mathbf{e}_{k+1}\right\|_{2}\right)$ (instead of $\sup \left(\left\|\mathbf{e}_{k+1}\right\|_{2}\right)$ in Theorem 4$)$ is calculated and the curve of $\max \left(\left\|\mathbf{e}_{k+1}\right\|_{2}\right)$ is illustrated in Figure 6. It is obvious that the values of both $\left\|\mathbf{e}_{k+1}\right\|_{2}$ and $\max \left(\left\|\mathbf{e}_{k+1}\right\|_{2}\right)$ are bounded as iteration number enlarges. 


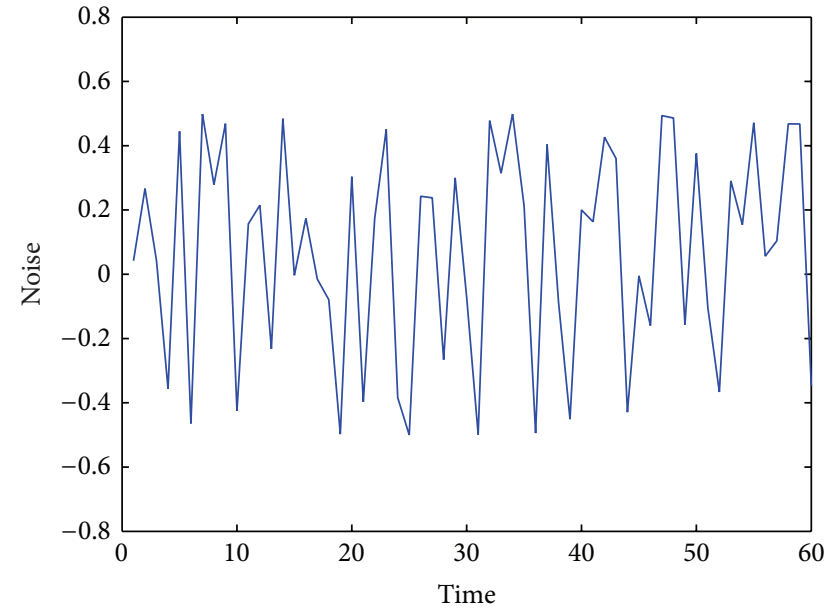

FIGURE 4: A sequence of random disturbance of arbitrary trial in time domain.

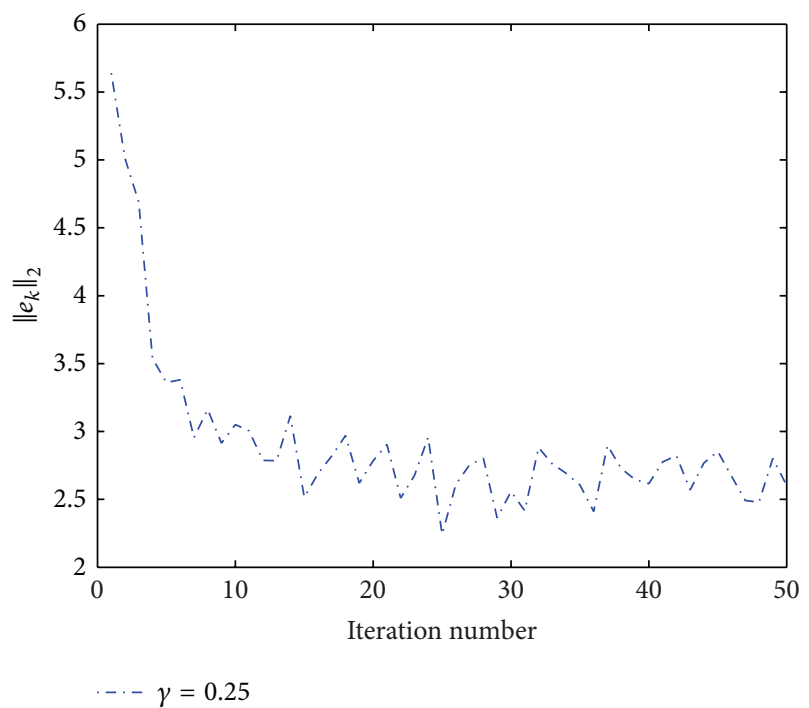

FIGURE 5: Tracking error in the iteration domain.

The system outputs at the 10th trial and the 15th trial are exhibited in Figure 7. It is observed that the tracking performance of the ILC algorithm is satisfactory when the switching rule is arbitrarily fixed and the system is disturbed by measurement noise with an acceptable boundary.

\section{Conclusion}

In this paper, a P-type ILC algorithm is applied to a kind of discrete switched systems with an arbitrary switching rule. By the super vector approach, the convergence performance is firstly discussed and then the robustness is analyzed when the system is randomly interfered by bounded measurement noise for the case that the switching rule is fixed once it is randomly selected for the first operation. Results manifest that the convergence and robustness can be guaranteed under appropriate conditions. However, for the case when

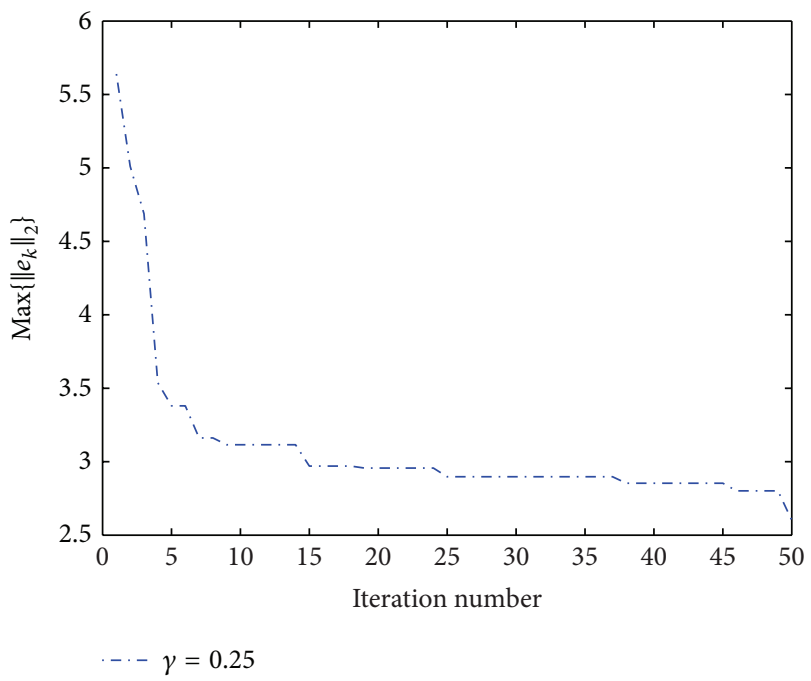

FIGURE 6: $\max \left\{\left\|\mathbf{e}_{k}\right\|_{2}\right\}$ profile.

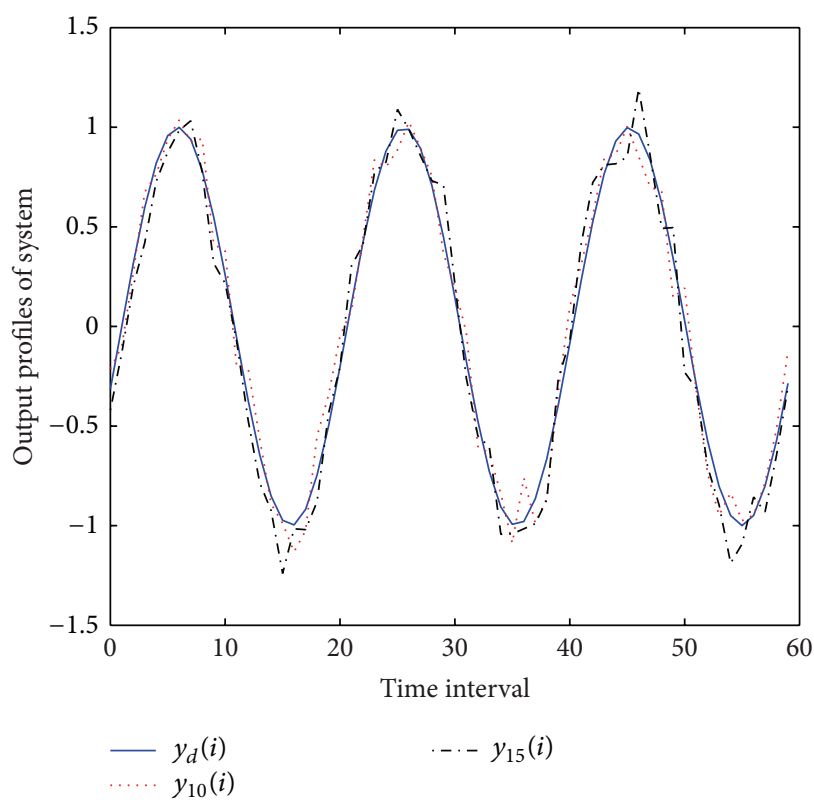

FIgURE 7: The system outputs of the 10th trial and the 15th trial.

the switching rule is fired randomly both in time axis and iteration direction, the analysis of the convergence and the robustness remain a hanging issue. In addition, the learning performance of the ILC scheme for linear timevarying switched system or nonlinear switched system is a challenging topic.

\section{Conflict of Interests}

The authors declare that there is no conflict of interests regarding the publication of this paper. 


\section{Acknowledgment}

The authors sincerely appreciate the supports of the National Natural Science Foundation of China under Grants nos. F010114-60974140 and 61273135.

\section{References}

[1] H. Lin and P. J. Antsaklis, "Stability and stabilizability of switched linear systems: a survey of recent results," IEEE Transactions on Automatic Control, vol. 54, no. 2, pp. 308-322, 2009.

[2] H. Lin and P. J. Antsaklis, "Asymptotic disturbance attenuation properties for uncertain switched linear systems," Nonlinear Analysis: Hybrid Systems, vol. 4, no. 2, pp. 279-290, 2010.

[3] M. C. Donkers, W. P. Heemels, N. van de Wouw, and L. Hetel, "Stability analysis of networked control systems using a switched linear systems approach," IEEE Transactions on Automatic Control, vol. 56, no. 9, pp. 2101-2115, 2011.

[4] Z. Ji, L. Wang, and X. Guo, "Design of switching sequences for controllability realization of switched linear systems," Automatica, vol. 43, no. 4, pp. 662-668, 2007.

[5] D. Cheng, "Controllability of switched bilinear systems," IEEE Transactions on Automatic Control, vol. 50, no. 4, pp. 511-515, 2005.

[6] J. P. Hespanha, D. Liberzon, D. Angeli, and E. D. Sontag, "Nonlinear norm-observability notions and stability of switched systems," IEEE Transactions on Automatic Control, vol. 50, no. 2, pp. 154-168, 2005.

[7] R. Wang, J. Xing, P. Wang, Q. Yang, and Z. Xiang, "Finite-time stabilization for discrete-time switched stochastic linear systems under asynchronous switching," Transactions of the Institute of Measurement and Control, vol. 36, no. 5, pp. 588-599, 2014.

[8] R. Wang, J. Xing, C. Zhou, P. Wang, and Q. Yang, "Finite-time asynchronously switched control of switched systems with sampled-data feedback," Circuits, Systems, and Signal Processing, vol. 33, no. 12, pp. 3713-3738, 2014.

[9] X.-H. Bu, F.-S. Yu, Z.-S. Hou, and F.-Z. Wang, "Iterative learning control for a class of linear discrete-time switched systems," Acta Automatica Sinica, vol. 39, no. 9, pp. 1564-1569, 2013.

[10] L. Zhang, P. Shi, and M. Basin, "Robust stability and stabilisation of uncertain switched linear discrete time-delay systems," IET Control Theory \& Applications, vol. 2, no. 7, pp. 606-614, 2008.

[11] X. Bu, F. Yu, and Z. Hou, "Iterative learning control for linear switched systems with arbitrary switched rules," in Proceedings of the 10th World Congress on Intelligent Control and Automation (WCICA '12), pp. 1182-1187, Beijing, China, July 2012.

[12] X. Bu, Z. Hou, F. Yu, and Z. Fu, "Iterative learning control for a class of non-linear switched systems," IET Control Theory \& Applications, vol. 7, no. 3, pp. 470-481, 2013.

[13] X. Ruan, Z. Z. Bien, and Q. Wang, "Convergence properties of iterative learning control processes in the sense of the Lebesguep norm," Asian Journal of Control, vol. 14, no. 4, pp. 1095-1107, 2012.

[14] H.-S. Ahn, K. L. Moore, and Y. Chen, Iterative Learning Control Robustness and Monotonic Convergence for Interval Systems, Springer, New York, NY, USA, 2007.

[15] X. Bu, F. Yu, Z. Fu, and F. Wang, "Stability analysis of highorder iterative learning control for a class of nonlinear switched systems," Abstract and Applied Analysis, vol. 2013, Article ID 684642, 13 pages, 2013. 


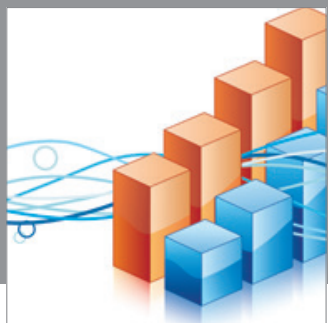

Advances in

Operations Research

mansans

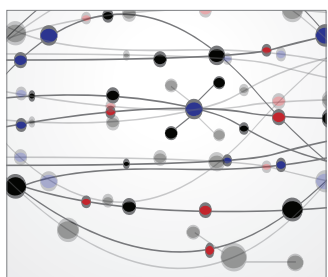

The Scientific World Journal
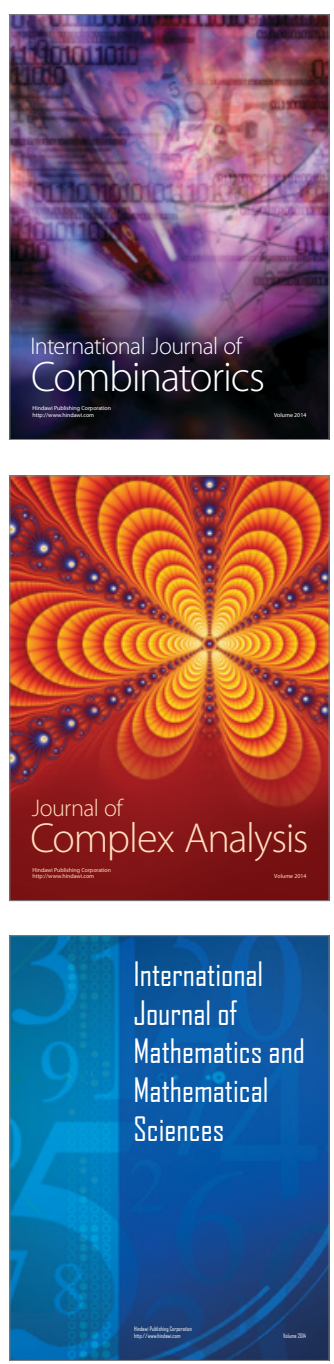
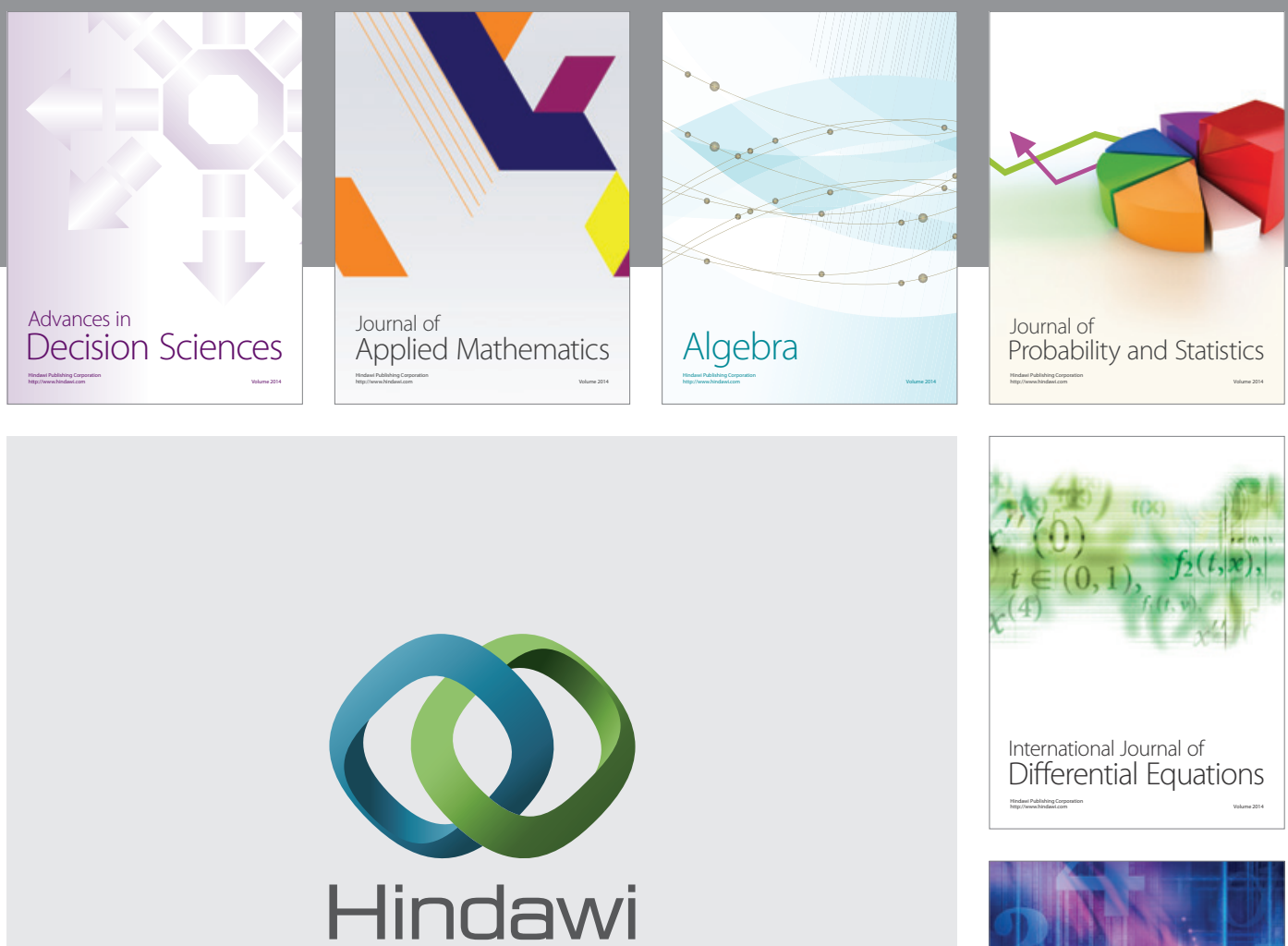

Submit your manuscripts at http://www.hindawi.com
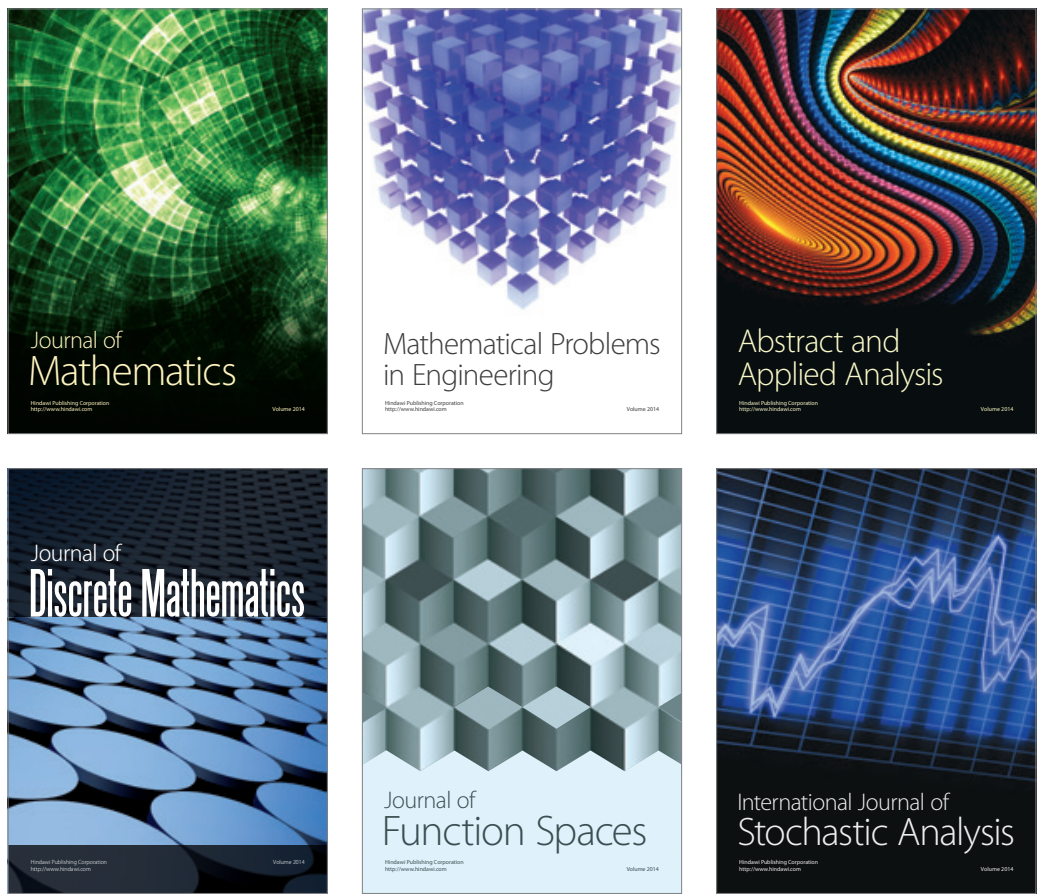

Journal of

Function Spaces

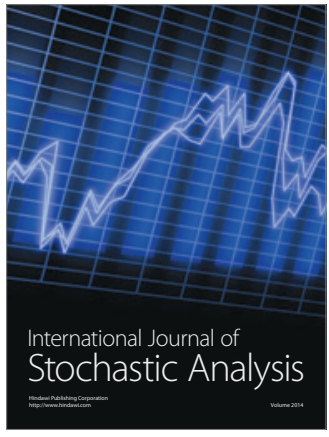

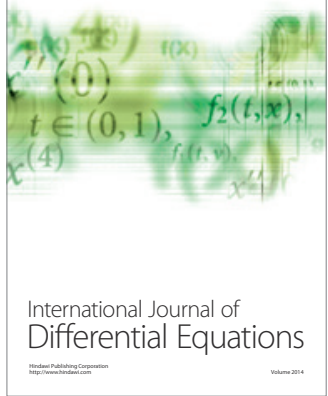
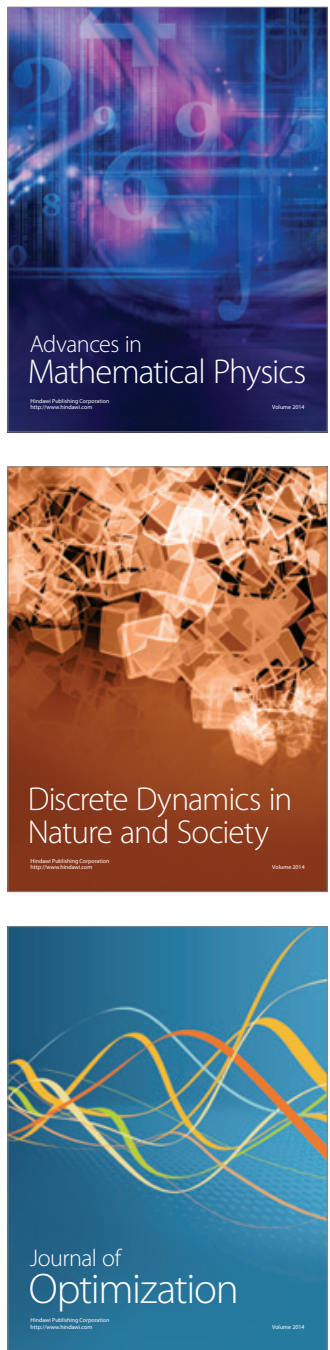